\title{
sciendo
}

\section{Logic in Natural Language: Commitments and Constraints}

\author{
Gil Sagi \\ University of Haifa \\ BIBLID [0873-626X (2020) 58; pp.277-308]
}

\begin{abstract}
In his new book, Logical Form, Andrea Iacona distinguishes between two different roles that have been ascribed to the notion of logical form: the logical role and the semantic role. These two roles entail a bifurcation of the notion of logical form. Both notions of logical form, according to Iacona, are descriptive, having to do with different features of natural language sentences. I agree that the notion of logical form bifurcates, but not that the logical role is merely descriptive. In this paper, I focus on formalization, a process by which logical form, on its logical role, is attributed to natural language sentences. According to some, formalization is a form of explication, and it involves normative, pragmatic, as well as creative aspects. I present a view by which formalization involves explicit commitments on behalf of a reasoner or an interpreter, which serve the normative grounds for the evaluation of a given text. In previous work, I proposed the framework of semantic constraints for the explication of logical consequence. Here, I extend the framework to include formalization constraints. The various constraints then serve the role of commitments. I discuss specific issues raised by Iacona concerning univocality, co-reference and equivocation, and I show how our views on these matters diverge as a result of our different starting assumptions.
\end{abstract}

\section{Keywords}

Formalization, logical consequence, logical form, normativity of logic, semantic constraints.

It is a common contention that natural language sentences or utterances have a logical form. In his recent book, Logical Form, Andrea Iacona formulates the following Uniqueness Thesis:

(UT) There is a unique notion of logical form that fulfils both the logical role and the semantic role. (Iacona 2018: 39) 
Iacona's own main thesis in the book is that (UT) cannot stand. The two different roles, to be discussed shortly, are in conflict and entail a bifurcation of the notion of logical form. While the semantic role of logical form requires an intrinsicalist notion of logical form (that logical form is determined by intrinsic properties of a sentence, e.g., its syntactic structure), the logical role runs counter to intrinsicalism. And while in a logically ideal language logical properties can be perceived "in the symbol alone" (Wittgenstein 1922, §6.113, in a sense to be qualified in what follows), natural language is riddled with vagueness, ambiguity and context sensitivity. Context, and more generally, factors external to a sentence, determine its logical properties.

I agree with Iacona that the notion of logical form plays different roles serving different purposes. However, while Iacona takes the project of assigning logical form to natural language sentences to be descriptive on either notion of logical form, I adopt a normative stance on the logical role. Contemporary logic studies logical properties through formal languages, and natural language sentences need to be formalized so that logical properties can be attributed to them systematically. This process includes a specification of a formal system, and an assignment of formulae to natural language sentences. That much is in agreement with Iacona. By contrast to Iacona, I suggest that both parts of the process have distinguished normative aspects. And so, the study of Iacona's new book serves the occasion to present an alternative approach and compare the implications of both.

In this paper, I thus focus on the logical role of logical form. I view formalization as involving various sets of commitments. One set of commitments concerns the rules of the formal system into which natural language sentences are formalized. Those rules may be those of standard predicate logic — but I propose a more general outlook, based on a model-theoretic framework I have proposed in (Sagi 2014), by which the choice of formal system is made by imposing semantic constraints on interpretations of expressions in the language. The assignment of formulae to natural language sentences, in turn, involves a set of formalization constraints. Both types of constraints express commitments on behalf of the theorizer.

The paper proceeds as follows. In $\S 1$, I discuss the logical and the semantic roles that Iacona ascribes to logical form. I tie these roles to two different projects logicians have engaged in in the $20^{\text {th }}$ 
century: the linguistic project, which studies natural language using formal tools, and the traditional project, which contributes to the methodology of science by providing formal tools for reasoning. The logical role of logical form is then connected to the traditional project, which in turn, has distinctive pragmatic and normative aspects. These aspects come to light when considering the notion of adequate formalization, which Iacona himself relates to logical form understood through its logical role. I discuss the motivation for formalization and its nature in $\S 2$, by considering the end result of formalization and the process itself. It will emerge that formalization involves spelling out explicitly rules by which arguments can be evaluated, which I analyse as explicit commitments made by a reasoner. In $\S 3$, I make formal sense of these commitments. In $\$ 3.1$, I utilize the model-theoretic framework of semantic constraints where a logical system is obtained by imposing constraints on models vis-à-vis the interpretation of terms in a given language. These constraints express the explicit commitments made by the reasoner. In addition, in $\S 3.2$, I define formalization constraints which express further commitments on the way natural language arguments are given a formal rendering which will be subject to semantic constraints. In $§ 3.3$, I discuss specific issues raised by Iacona concerning univocality, co-reference and equivocation, and I show how our views on these matters diverge as a result of our different starting assumptions. I conclude in $\S 4$.

\section{The logical and the semantic roles of logical form}

I take natural language to be a natural phenomenon to be studied empirically by the various sub-disciplines in linguistics. I shall speak of natural language sentences, but this is already at some level of abstraction from actual data. Linguistic occurrences, such as utterances, are concrete and are set in context. However, at different levels of abstraction, we can speak of different kinds of structures underlying utterances: phonetic, morphological, syntactic, semantic, etc. Logical form is presumably revealed at a high level of abstraction, and there the tools developed by logicians in the turn of the 20th century and onwards have found linguistic use. Formulas are used to represent logical forms of sentences in natural language. But what kind of properties are uncovered at this level of structure? 
Iacona contends that there are two distinct notions of logical form, serving different purposes. Logical form has been ascribed a logical role, as it is used in explaining logical properties and relations such as validity and contradiction. In addition, logical form has been ascribed a semantic role, as it is used in the formulation of a compositional theory of meaning (p. 37). ${ }^{1}$ There may be other roles that have been ascribed to logical form, but Iacona focuses on the two aforementioned to show that the same notion cannot fulfill all roles ascribed to it.

The semantic role, which Iacona associated with Frege and the tradition of formal semantics leaning on his work, is used to explain how the meaning of a sentence depends on the meanings of its parts. Iacona ties the semantic role to intrinsic properties of sentences, those which do not depend on external, contextual features. This is reasonable if we assume that compositional semantics is part of a theory of linguistic competence (Iacona speaks of the "conventions that are constitutive of a language" (pp. 66f) rather than linguistic competence - this difference will not matter much to what follows). Logical form, understood through its semantic role, is thus part of the subject matter of empirical linguistic study. Some ways of making sense of the intrinsicalist notion of logical form fulfilling the semantic role, according to Iacona, is through the notions of syntactic structure (as in Montague's work), LF (as in the Chomskian tradition), and semantic structure (as in the Davidsonian approach).

On the other hand, when it comes to the logical properties of sentences, an intrinsicalist approach does not suffice. Logical properties of sentences may vary with context, as Iacona illustrates by examples (p. 48) on which the following is based:

This is red and this is round

(*)

There is something that is both red and round

If this argument is uttered so that the two occurrences of "this" co-refer, then, at least prima facie, it is valid - otherwise it is not. So, the validity of the argument cannot be determined by its intrinsic properties.

${ }^{1}$ In what follows, all references by page number only are to Iacona 2018. 
Indeed, in some cases logical properties are not transparent to the reasoner, who is assumed to be linguistically competent. Those are cases where the reasoner, although linguistically competent, may still mistakenly believe that two occurrences of an expression refer to the same object (as "this" above, or a proper name used equivocally unwittingly (p. 72)). And so, if logical form on its logical role is to account for logical properties, it cannot be a matter of linguistic competence.

According to Iacona, the logical properties of a sentence are attributed to it vis-à-vis its content, which in turn is identified as what is said by the sentence and determines truth conditions. Logical properties, on Iacona's understanding of content, are determined by the truth conditions of the sentence (p. 53). The precise understanding of truth conditions and content are immaterial for Iacona's aim (though some clarifications are made, the details of which I shall not repeat). What is important is that, according to Iacona, the truth conditions of a sentence determine its logical form as it fulfils the logical role of explaining logical properties, and truth conditions require appeal to extrinsic properties of sentences. Now, since the semantic role requires an intrinsic notion of logical form, and the logical role requires an extrinsic notion, one and the same notion of logical form cannot fulfil both roles. I agree with Iacona's bifurcation of the notion of logical form for two purposes, but I offer an alternative take on the logical role of logical form, that has implications on what logical properties may be assigned to natural language sentences.

Both notions of logical form, according to Iacona, are essentially descriptive (p. 43). One describes semantic structure, and the other content. In what follows, I propose a different stance, which emphasizes logic's normative role. I reserve a descriptive notion of logical form for the scientific study of language. But I would also like to consider a notion of logical form that is not merely descriptive, which accounts for the use of logic by reasoners and interpreters. While the tools developed by logicians have found a fruitful application in linguistic study, the original aim of some of these logicians was very different. It would be useful to consider, for present concerns, different purposes for which logic has been used that are relevant to Iacona's distinction. 
Looking at work that has been done in the past century or so, we may distinguish between the linguistic project and the traditional project, both of which use logical tools. The linguistic project has natural language as its subject matter of investigation, and it employs formal tools of logic in its study. It has been advanced by 20th century authors such as Davidson, Harman, and Montague, as well as, arguably, Chomsky. Relevant to us here is that the linguistic project is properly descriptive. The traditional project, on the other hand, is methodological: it seeks to provide a tool for reasoning in science. We may consider Frege's Begriffsschrift as the paradigm of a contribution to the traditional project: "Its first purpose, therefore, is to provide us with the most reliable test of the validity of a chain of inferences and to point out every presupposition that tries to sneak in unnoticed" (Frege 1967: 6). The view of logic as subsumed under the methodology of science has been advanced by $20^{\text {th }}$ century authors such as Carnap, Tarski and Quine.

Historically, the aims of the two projects have often been intertwined: a better understanding of natural language can lead to a more successful use of it in reasoning. Certainly, one can contribute to both projects at once without conflict. But since the advent of formal languages, the two projects take on different routes. The availability of an alternative to natural language has brought revisionary tendencies to the traditional project, and in some cases to a complete break from natural language as a means for expression (as in Frege's Begriffsschrift) - and so also to a break from the linguistic project.

In both the traditional and the linguistic projects logical formalisms are used as a tool. But there is an important difference. In the linguistic project, a formal language may be used to describe or model linguistic phenomena. In the traditional project, logical formalisms are used as means for reasoning by a researcher whose subject of investigation may be that of any of the sciences. The linguistic role is descriptive, and the traditional role has distinctive normative aspects.

Despite the engrained normative aspects logic has on its traditional conception, some have denied that logic has a special claim for normativity. This has led to various defenses of the normativity of logic in the literature. A recent example is Steinberger (2019), who proposes three ways in which logic can be claimed to be normative. Logical norms provide first-personal directives that guide the reasoner; 
logical norms serve to make third-personal evaluations, and they play the role of third-personal appraisals (see Steinberger 2019: 7). These logical norms are paramount in the traditional project. I would like to suggest that the use of formal languages in the traditional project establishes a ground for these norms and provides a standard by which the fulfilment of these norms is measured. The articulation of a specified formal system to be used by a reasoner can be viewed as an explicit commitment to the rules of the system. Indeed, the rules provide directives guiding the reasoner, who commits him or herself to the evaluations and appraisals in light of the specified rules. We shall elaborate on this idea in the following sections.

Now, logical form serves different roles in either project. In the linguistic project, logical form is a theoretical concept that is used to explain linguistic phenomena. Indeed, this is precisely the semantic role discussed by Iacona. What role does logical form have in the traditional project?

Recall that our concern here is with the logical form of sentences in natural language, which the traditional project aims to revise and ideally replace by formal alternatives. But natural language may still be present and relevant to the traditional project. Despite the advance in formal tools, reasoners in various disciplines and contexts have not yet given up natural language. The practice of formalization serves to fill the gap between reasoning in natural language and the formal tools provided by logicians. In the process of formalization, formulae of a formal language are assigned to natural language sentences, so that their logical properties can be evaluated. This is where logical form plays a role in the traditional project: a formula assigned to a natural language sentence by a process of formalization represents the sentence's logical form. Arguably, logical form here plays the logical role alluded to by Iacona. However, by contrast to Iacona's position, logical form here is not a merely descriptive notion, but it is rather bound with the methodological and normative features of the traditional project. We shall discuss formalization in the next section. One of our main contentions will be that logical form is not discovered and described as in the work of linguists, but rather, it is assigned and committed to by agents as reasoners and interpreters.

Sure enough, Iacona has a different idea of the logical role of logical form in mind. Iacona speaks of logical form in its logical role as 
pertaining to objective notions of content and of truth conditions, that are there to be discovered and described. It may seem that while logical form in the linguistic project does match the semantic role Iacona ascribes to logical form, the normative notion of logical form that I associate with the traditional project is not the one Iacona aims at when he discusses the logical role - and therefore we may have three notions of logical form at hand (an option that Iacona seems to be open to (p. 38)). Be that as it may, it is worth noting that Iacona ties the logical role (and not the semantic role) of logical form to formalization. According to Iacona, "The truth-conditional view stems from the idea that an adequate formalization of a set of sentences provides a representation of their content" (p. 58). A formalization of a sentence, according to Iacona, mirrors its truth conditions relative to an interpretation, where an interpretation of a sentence fixes its content in accordance with its meaning (p. 59).

It is clear that while there may be principles guiding adequate formalization, the end result is highly underdetermined. Moreover, while the adequate criteria of formalization and the nature of formalization itself have been in dispute, there seems to be wide agreement among writers that formalization is not a merely descriptive matter. According to some, formalization is a form of explication, and it involves normative, pragmatic, as well as creative aspects. If Iacona's discussion of formalization is aimed at the same practice referred to by these writers, then there is room to doubt that the notion of logical form which he aims at, which fulfils the logical role, is merely descriptive. In the next section, I present an approach to formalization that will provide an alternative, normative outlook to the logical role of logical form.

\section{Formalization as a humanistic endeavor}

By "formalization" one can refer to a process or to an end result. The end result consists of sentences or arguments in a formal language, perhaps with some kind of dictionary. The process takes sentences or arguments in natural language and produces a formal rendering. In fact, as explained by Brun (2014), formalization is a stage in a wider process of argument reconstruction, where a text in a natural language is analysed and is evaluated with respect to the validity of the in- 
formal arguments it contains. This process includes a stage of argument analysis, producing informal inferences where the premisses and conclusions are spelled out, followed by formalization, which produces formal arguments which can be assessed by the rules of a formal system.

Let us first consider the end result of formalization. We have mentioned that Frege's Begriffsschrift is aimed at providing a "most reliable test of validity" (Frege 1967: 6). Natural language, while useful for everyday purposes, is ill-suited for this task. Ambiguity, vagueness and context-sensitivity are just a few of the phenomena that obstruct the use of natural language for scientific purposes. A language that is suitable for scientific purposes, where validity is paramount, will minimize fallacies that originate in language use. Such a language will be maximally logically perspicuous. Wittgenstein expressed this Fregean objective as a feature of the Tractarian ideal language, where "It is the characteristic mark of logical propositions that one can perceive in the symbol alone that they are true; and this fact contains in itself the whole philosophy of logic." (Wittgenstein 1922, §6.113). Logical propositions correspond to nothing in reality, so their truth depends merely on the symbols. More generally, the ideal, using Iacona's terminology, is that logical properties would depend only on intrinsic properties of sentences.

Carnap has added a qualification to Wittgenstein's remark. Symbols on their own do not have logical properties: those are acquired only in a linguistic framework. Carnap remarked that "It is certainly possible to recognize from its form alone that a sentence is analytic; but only if the syntactical rules of the language are given." (Carnap 1937: 186). Carnap's addition that the rules must be given comes from his position that there is a conventional aspect to logic. At this stage in his work, Carnap speaks of syntactic rules (which include rules of inference and of well-formedness), but the point generalizes to semantic or other frameworks as well. Logic is relative to a linguistic framework, so only given a framework can analyticity (or logicality) be determined.

Whether or not logic is conventional in the way Carnap thought it was, symbols certainly have no logical properties on their own - it is only in virtue of their meaning or the rules governing them that a logical property can be attributed to them. And so, when formalizing 
into an artificial language, one must specify its rules. And setting the rules, or choosing a formal system into which to formalize, is not a trivial matter.

So even in an ideal language, logical properties cannot be recognized from the symbols alone (if the ideal should be conceivably attainable). Rules pertaining to the symbols are involved as well. Presumably, there are rules governing the use of natural language expressions too. If our interest is in evaluating arguments in natural language, we can undertake the task of exposing these rules through linguistic analysis, and use them to make an assessment - what is then the gain in the use of a formal language?

First, the most obvious gain is that of decontextualization. Natural language sentences are stated in context, and as argued by Iacona, their logical properties require an extrinsic notion of logical form. And so, even the symbols together with the rules do not suffice to determine logical properties: context is another non-trivial factor. Context resolves structural and lexical ambiguity, as well as the coreference or lack thereof of demonstratives, as in example $(*)$. The outcome of formalization is phrased in a context-insensitive formal language. This still leaves us apart from the Tractarian ideal, but it brings us to the Carnapian more realistic goal: the logical properties in a decontextualized language depend on the symbols and the rules alone. $^{2}$

Secondly, and relatedly, formalization gives another important benefit. While both natural and formal language are governed by rules, only in a formal language the surface structure reflects the logical form of the sentence, and so by formalizing the logical properties are made perspicuous. In natural language, one is more likely to be misled by the surface structure. Formal languages, by virtue of their perspicuity, provide means for "calculating" logical properties from the surface structure. Moreover, it has been proposed that

\footnotetext{
${ }^{2}$ Context-sensitivity, or at least some aspects thereof, have been incorporated into the logical treatment of natural language, as in Kaplan 1989. For recent treatments of logicality in a context-sensitive setting, see Woods 2017 and Woods and Michaelson, manuscript. These studies are of significance for understanding logical form in its semantic role, as they facilitate an account of logicality that is based on speakers' competence. How these studies may relate to the traditional aim, which is at issue here, is a matter we shall not pursue.
} 
formal languages serve as "cognitive artefacts enhancing and modifying an agent's reasoning processes" (Dutilh Novaes 2012).

Nonetheless, I suggest that the significance of formalization goes well beyond decontextualization and the unification of surface structure and logical form. Recall that formalization here is conceived as a stage in argument reconstruction. This process is carried out by a reasoner or an interpreter, and it ultimately serves for the evaluation of logical properties of a given text. By moving to a formal language, the reasoner makes explicit their interpretive and logical commitments: what they take to be the form of the arguments of the given text, and what logical rules they subscribe to in its evaluation. ${ }^{3}$ Formalization thus provides the ground for logical norms to be applied.

In this connection, the transition to a formal language is not a merely mechanical procedure. The view of formalization that emerges takes natural language sentences not merely as objects of formal study, but also as vehicles of reasoning to be interpreted and evaluated. The analysed text is treated as the expression of an interlocutor. The idea that a text can be thoroughly interpreted and evaluated using formal means makes formalization a humanistic endeavor par excellence. In the rest of this section we spell out some implications and further aspects of this approach.

It would be helpful to say a few words about the process of formalization. It is generally agreed among authors that even though the process of formalization is far from trivial, there are basic criteria of adequacy. An adequate formalization should be in line with judgements of informal validity (Baumgartner and Lampert 2008). Making this idea precise, however, brings with it disagreement among authors. When formalizing, should one appeal to the most charitable interpretation of the text, and e.g. add hidden premisses to make stated arguments valid, or should one try to track the author's intention whether felicitous or not, or neither? This issue is related to a more general concern: should the process of formalization involve

\footnotetext{
${ }^{3}$ The view presented here should be distinguished from that of Brandom 1994. While making explicit here and in Brandom exposes inferential relations and allows them to be accessible to critical discussion, in the present context making explicit means spelling out rules in the context of a formal system, whereas Brandom speaks of making inferential relations explicit using logical vocabulary within a language. For a critical discussion, see Brun 2018.
} 
some kind of reflective equilibrium (following Rawls (1999) and Goodman (1983)) between judgments of instances, general rules and perhaps a background theory? Resnik (1985), Peregrin and Svoboda $(2013,2017)$ and Brun $(2014,2017)$ are examples advocating reflective equilibrium, and Thagard (1982) and Baumgartner and Lampert (2008) are in opposition. Should criteria for adequate formalization be semantic (Sainsbury 1993; Baumgartner and Lampert 2008), or be based on inferential grounds (Peregrin and Svoboda 2013, 2017)? Should findings from empirical linguistics or psychology have substantial impact on logical practice? Thagard (1982) is for, and Resnik (1985) is against.

These are just a few of the points of contention, without aiming at a full literature survey. In what follows I shall not take a stand on specific criteria for adequate formalization. My aim is to characterize the process as a whole, and to provide a formal framework. At this point, we may observe that formalization is treated in the literature not as a merely descriptive endeavor, and that it involves normative and pragmatic concerns. If the purpose of the formalization is to evaluate the logical validity of arguments presented in some text (rather than a purely linguistic study of the text), the task includes interpretive and exegetical aspects. Indeed, authors have emphasized the creative aspects of formalization, some going so far as claiming that there is no hidden logical form that is exposed by formalization (Brun 2014; Peregrin and Svoboda 2017).

Carnap was one of the main advocates of the use of formal tools in philosophy and science. The method of Carnapian explication takes an informal concept (the explicandum), and replaces it with one that is more precise (the explicatum) - ideally with a formal one couched in a formal system. Explication has four main desiderata: similarity of the explicatum to the explicandum, exactness of the rules of use of the explicatum, fruitfulness, and simplicity (Carnap 1962: 5-8). The explicatum will not have the same meaning as the explicandum, nor even the same extension, except in distinguished contexts. Explication is a pragmatic endeavor, and its aim is methodological: to enable scientific progress by the introduction of more precise concepts.

Several authors have adopted an approach to formalization that is akin to Carnapian explication, specifically as in both we move from 
the informal to the (more) formal and exact. For example, according to the view advanced by Baumgartner and Lampert, "Formalization, thus, is a means to explicate informal reasoning... formalizations are not and will never be the result of a formal, effective formalization procedure, because formalizing a natural language text $\mathcal{T}$ crucially rests on an informal understanding of $\mathcal{T}$ and not simply on its surface grammar" (Baumgartner and Lampert 2008: 25). I am in agreement with this approach, but further, I subscribe to the dominant pragmatic aspects of Carnapian explication: a formalization does not aim at an accurate translation, nor does it reveal a hidden logical form. According to Brun, "In sum, formalizing is not merely abstracting but also involves creative and normative aspects of constructing logical forms and resolving matters of logical form, which are alien to paradigmatic forms of translation." (Brun 2014: 102) When formalizing we do assign to sentences a logical form, but this assignment is not made on a merely descriptive basis.

Formalization, as it has been presented, involves making choices: choosing a formal system and its rules and choosing which formal expression to assign to which natural language expression. These choices are guided, at least in part, by the desiderata for Carnapian explication. But what I take to be of primary importance in this process, is that the rules are stated explicitly, and they are thus committed to and thereby endowed with normative force. What marks the difference between formal language and natural language is precisely this: a formal language is constituted by explicitly stated rules. While natural language might be governed by rules, those are not given explicitly at the outset, and they need to be discovered by linguistic study on the basis of recorded regularities. The outcome of linguistic study is subject to correction and revision, while the rules of a formal language cannot be erroneously attributed to the language they themselves define.

The explicitly stated rules of a formal language are constitutive of it, and their status as such is the source of their normative force. When employing a formal language, one is subject to evaluation visà-vis its rules. Indeed, when one uses a formal language, one thereby commits oneself to the norms of following its rules. ${ }^{4}$ It is important

${ }^{4}$ How these norms, that are derived from the rules, are spelled out exactly is 
that the rules are explicit, because only then can one properly commit to them and be evaluated according to them. Natural language is a complex, dynamic, natural phenomenon. When we formalize, we rigidify language (see also Peregrin and Svoboda 2017: 3). Once the rules are set explicitly and committed to, there is no recourse to previous use. Surely, the rules can be revised, but that again must be done explicitly and it entails a renouncement of previous commitments.

\section{Formalization and semantic constraints}

In this section, I would like to explore the ideas of the previous sections in a formal implementation. We shall look at a model-theoretic framework of semantic constraints which is a generalization of standard first order logic (Sagi 2013, 2014).

In standard, first- or higher-order logical systems, a distinction is made between the logical and the nonlogical vocabulary. The logical vocabulary gets a fixed meaning over models, and the nonlogical vocabulary gets any interpretation that accords with its semantic category. Standardly, the logical vocabulary includes the propositional truth-functional connectives, the existential and universal quantifiers, and perhaps the identity sign as well. However, the question of whether there is a principled distinction between logical and nonlogical terms has occupied philosophers of logic in recent decades. Various criteria have been proposed — invariance criteria (which shall be mentioned in §3.1) are perhaps the most widely accepted. Some, on the other hand, have been skeptical about there being a principled distinction between logical and nonlogical terms, and have offered relativistic or pragmatic outlooks.

In the previous section, I suggested that in the context of formalization, the rules of a formal system express commitments on behalf of the reasoner, interpreter or interlocutor. In standard systems, these rules will include the recursive syntactic and semantic clauses for expressions of the language. Specifically, one commits to a fixed interpretation of the logical vocabulary, and makes no commitment with respect to the nonlogical vocabulary, apart from the division

not a trivial matter. For this issue, I refer the reader to Steinberger 2019. 
into semantic categories. The nonlogical expressions may have associated natural language counterparts, but after formalization the latter's meaning is completely abstracted away. However, when it comes to the analysis of arguments, this mode of operation might be unduly stringent. There may be cases where it is best to fix the interpretation of a term to some extent without fixing it completely as a logical term. One may wish not to commit to the interpretation of a connective in all cases, e.g. leave it open whether a disjunction is inclusive or exclusive; or to commit to a pair of terms having the same interpretation without committing to what that interpretation is; or, not to commit to the semantic category of a term but only to that of expressions containing it.

In previous work, I have proposed a model-theoretic framework of semantic constraints which allows fixing the interpretation of terms in various ways and to different degrees. No division of the vocabulary into semantic categories, nor its division into the logical and the nonlogical is assumed at the outset. The interpretations of terms are restricted through constraints on models used to interpret the language. This framework is a generalization of standard first order logic, as all the standard rules can be rephrased as semantic constraints. The framework can also be extended to higher-order or intensional semantics. In the next subsection, I give the basic details of the extensional framework.

The end result of formalization, on the present approach, is a system of semantic constraints to which there is an explicit commitment. However, formalization includes further commitments that have to do with the transition from natural language to a formal language. In $\S 3.2$ I shall thus add to the framework the notion of formalization constraints, which will express these further commitments.

\subsection{Semantic constraints: the framework}

This section presents the basics of the framework of semantic constraints. We start with some definitions, give some examples, and at the end discuss the considerations on the basis of which a set of semantic constraints may be chosen and committed to.

First, we need to say what a language consists of and how it is interpreted. Both what is considered a language and what is considered 
a model for the language are generalized and include very little assumptions at the outset. The syntax we assume for a language is very minimal, and includes two basic categories. A language L consists of terms (the primitive expressions) and phrases (the complex expressions). Phrases are strings of terms, perhaps with the addition of auxiliary symbols such as parentheses (every term is a phrase, but not every string is a phrase). No rules of well-formedness other than the specification of phrases are assumed at the outset.

A model for $\mathrm{L}$ is a pair $\mathrm{M}=\langle\mathrm{D}, \mathrm{I}\rangle$ such that $\mathrm{D}$, the domain, is a nonempty set, and I is an interpretation function. Since we do not assume clauses for logical vocabulary at the outset, nor do we assume compositionality of the language, the interpretation function is therefore unconstrained at the outset. An interpretation function is thus a function that takes phrases to elements in the set-theoretic hierarchy built over $\mathrm{D} \cup\{\mathrm{T}, \mathrm{F}\}$ (the domain and the truth values).

The metalanguage includes, apart from names for the expressions in the object language, the language of set theory with optional additional vocabulary depending on the semantic constraints to be formulated. A semantic constraint is a statement in the metalanguage that includes an implicit quantification over models, domains and interpretation functions, that somehow constrains or restricts the admissible models for a given language. All standard semantic clauses for the interpretation of logical vocabulary can be rephrased as semantic constraints, which may or may not be included in a system, as well as a host of other kinds of constraints. ${ }^{5}$

So, for example, the following statement regarding the interpretation of conjunction, is a semantic constraint suitable for propositional logic: ${ }^{6}$

$$
I(\phi \wedge \psi)=T \Leftrightarrow I(\phi)=I(\psi)=T
$$

${ }^{5}$ Semantic constraints are akin to meaning postulates (expressed in the metalanguage) as in the work of Carnap and Montague. The essential difference is that in the work of these authors, meaning postulates come on top of a logical system constituted by recursive clauses for the logical terminology. Here, there is no fundamental difference between clauses for logical terms and other semantic constraints, and there is no underlying logical system assumed (see Sagi 2014: 267f).

${ }^{6}$ Predicate logic requires an adjusted condition in terms of satisfaction (see Sagi 2013). 
A model admissible by this constraint is such that it interprets conjunction in the standard way. Other semantic constraints may constrain the mutual interpretation of terms, for example:

$$
I(\text { allRed }) \cap I(\text { allGreen })=\varnothing
$$

and

$$
I(\text { even }) \cap I(\text { odd })=\varnothing
$$

rule out models where the intersection of allRed and allGreen or of even and odd are non- empty. More examples constraining set-theoretic relations may include:

$$
\begin{aligned}
& I \text { (bachelor) } \subseteq I(\text { unmarried }) \\
& I \text { (wasBought })=I(\text { wasSold })
\end{aligned}
$$

The language is not assumed to have semantic categories at the outset. The following constraints serve the purpose of restricting the interpretation of a term to some standard semantic category:

$$
I(J o h n) \in D
$$

restricts John to be always interpreted as a member of the domain,

$$
I(b i g) \subseteq D
$$

restricts big to be interpreted as a subset of the domain, and

$$
I(a b c) \in\{T, F\}
$$

restricts the phrase $a b c$ (the string composed of the terms $a, b$ and c) to be interpreted as a sentence: to always take a truth value as its interpretation.

One can also partially fix the interpretation of a term by demanding that some object would be a member of it (in which case that object is thus forced to be a member of every domain to be considered admissible), for example by:

$0 \in I$ (naturalNumber)

Here the number 0 is forced to be a member of the interpretation of naturalNumber. This should be distinguished from a constraint that forces the interpretation of a term $\mathbf{0}$ to be a member of the interpre- 
tation of naturalNumber: ${ }^{7}$

$$
I(\mathbf{0}) \in I(\text { naturalNumber })
$$

In all these examples, we have statements in the metalanguage regarding the interpretation of expressions in the object language which may be viewed as expressing commitments with respect to the object language. The semantic constraints above need not all be endorsed in a system for logic_-various considerations are in place when choosing which constraints to finally commit to-we shall discuss some of them in what follows.

Given a set of semantic constraints $\Delta$, a class of the $\Delta$-admissible models is determined. While the initial class of models makes no distinctions between different terms and phrases, the more semantic constraints are included, the more distinctions can be made. Several semantic notions can be defined in the framework. Logical validity (w.r.t. $\Delta$ ) is defined as truth preservation over the $\Delta$-admissible models. For definitions of other notions (dependency, determinacy, compositionality etc., see Sagi 2014).

The main value of the framework of semantic constraints is in its generality and in that it makes only minimal assumptions made at the outset. No strict distinction between logical and nonlogical vocabulary needs to be assumed (though such a distinction can be accommodated). This generality is relevant to our present concern as well. Different features of logical systems in this framework are expressed as statements that may or may not be endorsed, and in the present context these are explicit commitments that may or may not be made with respect to the language.

The framework of semantic constraints can be used descriptively, e.g. in the study of certain features of natural languages. Here we are considering semantic constraints as providing the end result of formalization, and so, from the perspective of a reasoner, an interpreter or an interlocutor. A set of constraints is chosen, relative to which validity is defined and arguments are assessed. The chosen constraints can be the outcome of an explication of expressions in the source language from which arguments have been formalized,

\footnotetext{
${ }^{7}$ In the case of numerals, I use boldface to distinguish the numeral as a term in the object language.
} 
in line with the view of formalization presented in the previous section. However, the guiding principle on which this choice is based, I suggest, is whether the reasoner (interpreter, interlocutor) would commit to the given semantic constraints. Are these constraints appropriate to set the standard by which the formalized arguments should be evaluated?

Two sets of considerations are then in place. The first set of considerations revolves the question, is the given constraint adequate with respect to the meanings we would like to capture? For example, is the example constraint above regarding conjunction adequate with respect to the connective meaning we would like to capture? Is it adequate to demand that the extensions of allRed and allGreen be mutually exclusive, given the meanings that they are intended to capture? Here we may include all the considerations that take place when carrying out an explication. We may wish to remain close to usage of terms in the source language, and thus include empirical descriptive adequacy with respect to that language as a significant factor. Or, we may consider the simplicity and fruitfulness of the candidate semantic constraint as decisive factors.

The second set of secondary considerations revolves the question, is the given constraint adequate for being included in a system for logic? It might be that we have good reason to explicate naturalNumber so that its extension will include 0 , or allRed and allGreen so that their extensions are mutually exclusive. Yet, these reasons might not suffice for including the relevant semantic constraints in a system for logic - it might be that these features of these terms' meanings is not something that we would like to include in a system deciding logical validity. If we have a criterion for the logicality of semantic constraints, it might deem some constraints as unacceptable regardless of their adequacy with respect to intended meaning. Let us elaborate some more on the consideration of logicality.

We have mentioned that the strict distinction between the logical and nonlogical vocabulary in standard systems raises the question of whether a criterion could be formulated on the basis of which the distinction could be made. While the framework of semantic constraints forgoes the strict distinction, the question of a criterion remains in a different form. In the more general framework, terms can be fixed in a variety of manners - but how should they be fixed? 
Is there a criterion for the "correct", or the "logical" semantic constraints? It would take us too far afield to deal properly with the issue of criteria for the logicality of semantic constraints. ${ }^{8}$ Relevant to us here, is that if such a criterion is accepted, its function in formalization as we approach it is that of a meta-commitment. While each semantic constraint accepted expresses a commitment with respect to the interpretation of the object language, a criterion for semantic constraints expresses a second level commitment that can be applied to any object language treated in the framework. Such a criterion thus concerns the framework itself and how it may be used, and can be used to express a commitment with respect to the semantic apparatus itself employed in the formalization.

Finally, the system of semantic constraints that serves as the end result of formalization embodies a set of explicit commitments that follow from a range of considerations: some having to do with the semantic apparatus used, some with descriptive aspects of the source language, some with the potential fruitful use of the system on different occasions. Indeed, the end result can be seen as explicating, or as partially explicating the terms that are constrained. And once a system of constraints is formulated explicitly, it is normatively binding. While an argument given in natural language can be described and interpreted in different ways and its logicality is always underdetermined, this is not so when commitments with respect to the language are made explicit. Any argument formulated within a formal system whose rules are stated explicitly is subject to evaluation, appraisal or blame, on the basis of the commitments made by the statement of the rules - and in the present proposal - the rules are rules of interpretation in the form of semantic constraints.

\subsection{Formalization constraints}

We should now deal with the process of formalization: with the transition from an argument in natural language to an argument in

\footnotetext{
${ }^{8}$ In work in progress, I propose a meta-constraint which serves as a necessary condition on the inclusion of a semantic constraint in a system for logic. The condition is a generalization of the criterion of invariance under isomorphisms for logical terms.
} 
a formal language. Recall that the process of formalization decontextualizes and gives to each natural language argument a formal rendering that is governed by explicitly stated rules. In the previous section, we have not specified a specific formal language with a set of rules - we have only presented a framework in which such a system can be defined through semantic constraints. Accordingly, in this section, we shall only delineate the notion of a formalization constraint. Specific formalization constraints are chosen ad hoc on a particular occasion of formalization. Formalization constraints express explicitly stated commitments on behalf of the formalizer with respect to the formal rendering of a given text.

Formalization is always carried out with respect to a particular text in context. We may thus conceive of a formalization function that takes sequences of source language expressions in context as arguments, and gives target language expressions as values. Such a sequence can be anything from a single expression in context to the sequence consisting of the whole text to be analysed.

A formalization function is ad hoc in that it is limited to a given text to be analysed on an occasion. To allow for various kinds of context shifts, we do not assume a constant context throughout the given text. Each source language expression is given in some context, and we do not assume at the outset that any pair of contexts involved are equal, nor that they are distinct.

So for a formalization function $F$, we assume that the domain of $\mathrm{F}$ is $\left.\left\{<<e_{1}, c_{1}\right\rangle, \ldots,<e_{n}, c_{n}\right\rangle>$ : for each $1 \leq i \leq n, e_{i}$ is a source expression, $c_{i}$ is a context, and $\left.n \in \mathrm{N}\right\}$ and the range of $F$ is the set of phrases of the target language. Ordinarily, there will be systematic relations between the values of sequences and those of the subsequences consisting them (e.g. if the source language is assumed to be compositional), but those are not forced at the outset.

In particular examples, instead of a pair of an expression and a context, we shall attach a subscript to an expression to designate the context in which it is expressed: this $s_{i}$ is the occurrence of the expression "this" in context $i$. It should be noted that expressions in context already pose some level of abstraction from the way in which natural language sentences are actually presented to us. Normally, an argument is presented through a concrete occurrence of speech or writing, from which expression-context pairs can be extracted through 
abstraction. This process is important to note, because it involves the nontrivial decision of whether two concrete speech or text occurrences, while being distinct, share either or both parameters of linguistic expression and context. For instance, this stage of abstraction decides whether two occurrences of a proper name each referring to a different individual, are considered as different linguistic items, or as the same expression used in different contexts.

The target language consists of terms and phrases governed by a set of semantic constraints. As for the source language, we assume that it consists of expressions, primitive and complex, in which we distinguish only a category of declarative sentences. We may have an elaborate theory of the source language, but we are not assuming that such exists at the outset. The formalization function assigns to each occurrence of a declarative sentence in the source language (construed as a sequence of expressions in context) a sentence in the target language, ${ }^{9}$ so that the outcome of a formalization of a source language argument is a target language argument that can be assessed for validity. The formalization function may be partial, except when restricted to the occurrences of declarative sentences in the source text.

We are thus given a sequence of natural language expressions in context. We assume that some argument analysis has been done to discern the premisses and the conclusion. Beyond that, we assume as little as possible on the outcome of formalization at the outset - we use formalization constraints to narrow down possible outcomes.

An occurrence of a declarative sentence in the source language will normally span over different contexts, if it is composed of expressions which in turn are expressed in different contexts. Recall example (*) (p. 4), where two occurrences of "this" may be used to denote different objects in the very same sentence: this is possible only when interpreting each occurrence of "this" relative to a different context.

Now, it has been noted that any useful formalization cannot be a one-one function from expression-context pairs to target language expressions. In such a case, there will be no repetition in the text, and

${ }^{9}$ Recall that in the framework of semantic constraints, a division of phrases into grammatical categories is not assumed at the outset: sets of constraints impose the relevant distinctions. So, for instance, we may define a sentence as follows: A phrase $s$ is a sentence (with respect to a set of semantic constraints $\Delta$ ) if $\mathrm{I}(s)=\mathrm{T}$ or $\mathrm{I}(s)=$ F in every $\Delta$-model. 
we lose all possibility of a pattern in an argument that can give rise to interesting logical properties. For example, assume the target language is that of standard, propositional logic. The formal argument:

$$
\frac{p \wedge q}{q}
$$

would never be the outcome of formalization, because it would never be the case that the same propositional letter is used twice (Brun 2008: 7). ${ }^{10}$

The role of formalization constraints is thus to expose patterns in the source text. This can be done by either forcing the formalization function to take multiple source expressions to some specific target expression:

(1) $\mathrm{F}\left(<I t_{c_{1}} i_{c_{2}}\right.$ raining $\left.c_{c_{3}}>\right)=p$

(2) $\mathrm{F}\left(<I t_{c_{4}} i_{c_{5}}\right.$ raining $\left._{c_{6}}>\right)=p$

or by forcing the formalization function to give the same value to two occurrences of a source expression, without forcing a specific value:

(3) $\mathrm{F}\left(<I t_{c_{1}} i_{c_{2}}\right.$ raining $\left._{c_{3}}>\right)=\mathrm{F}\left(<I t_{c_{4}} i s_{c_{5}}\right.$ raining $\left._{c_{6}}>\right)$

By the latter constraint (3), we are making an explicit commitment to the effect that two utterances of "it is raining" will be formalized into the same phrase and thus obtain the same value in every model for the target language. By the preceding pair of constraints, (1) and (2), we are making a commitment to a specific formalization, a consequence of which is that they are formalized into target phrases which are given the same value by the interpretation function in every model.

Analogously, we may constrain the formalization function to take two source expressions to different phrases. This can be done by

\footnotetext{
${ }^{10}$ We may note that the framework of semantic constraints is general enough to accommodate systems where validities do not include any repetition (e.g. by employing semantic constraints that force different expressions to be assigned the same interpretation, see the discussion of co-reference in $\S 3.3$ ), but such systems would be extremely cumbersome and would exclude any of the standard logical validities.
} 
directly formalizing into distinct phrases:

(4) $\mathrm{F}\left(\right.$ this $\left._{c_{77}}\right)=a$

(5) $\mathrm{F}\left(\right.$ this $\left._{c_{8}}\right)=b$

or by just constraining the formalization function to assign to the two occurrences different phrases:

(6) $\mathrm{F}\left(\right.$ this $\left._{c_{7}}\right) \neq \mathrm{F}\left(\right.$ this $\left._{c_{8}}\right)$

Note that the target expressions might still be given the same interpretation in a model, if there is no semantic constraint forbidding it, such as

(7) $I(a) \neq I(b)$

A fortiori, there may be a semantic constraint forcing the two phrases to be given the same value in every model:

(8) $I(a)=I(b)$

Finally, the formalization function will take some expression occurrences to designated phrases in the target language that have specified semantic constraints restricting their possible interpretations, as those mentioned in the previous section:

(9) $\quad \mathrm{F}\left(a_{\mathrm{nnd}}\right)=\wedge$

(10) $\mathrm{F}\left(\right.$ red $\left._{c_{10}}\right)=$ allRed

(11) $\mathrm{F}\left(\mathrm{red}_{c_{11}}\right)=$ allRed

The examples of formalization and semantic constraints we have given so far (either (1) and (2), or just (3)) enable us to ascertain the validity of the following argument, if we include the semantic constraints $\mathrm{I}(p) \in\{\mathrm{T}, \mathrm{F}\}$ and $\mathrm{I}(q) \in\{\mathrm{T}, \mathrm{F}\}$, and a constraint to the effect that the formalization of the premise is a concatenation of the formalization of its parts:

$$
\frac{I t_{c_{1}} i s_{c_{2}} \text { raining }{ }_{c_{3}} \text { and } d_{c_{9}} i t_{c_{12}} i s_{c_{13}} \text { snowing } c_{c_{14}}}{I t_{c_{4}} i s_{c_{5}} \text { raining }_{c_{6}}}
$$


By the possible formalization:

$$
\frac{p \wedge q}{p}
$$

And the same constraints enable us to ascertain the invalidity of the following argument, on the condition that we do not include the semantic constraint $I(a)=I(b)$ (or constraints that entail it), and that we include in addition the semantic and formalization constraints $\quad \mathrm{F}\left(\right.$ This $\left._{c_{7}} i s_{c_{15}} \operatorname{red}_{c_{10}}\right)=\operatorname{allRed}(a), \quad \mathrm{F}\left(\operatorname{This}_{c_{8}} i s_{c_{16}} \operatorname{red}_{c_{11}}\right)=\operatorname{allRed}(b)$, $\mathrm{I}(\operatorname{allRed}(a)) \in\{\mathrm{T}, \mathrm{F}\}, \mathrm{I}(\operatorname{allRed}(b)) \in\{\mathrm{T}, \mathrm{F}\}:$

$$
\text { This }_{c_{7}} \text { is } c_{c_{15}} \mathrm{red}_{c_{10}}
$$

$\operatorname{This}_{c_{8}} i s_{c_{16}} \operatorname{red}_{c_{11}}$

By the possible formalization:

$\operatorname{allRed}(a)$

$\operatorname{allRed}(b)$

Formalization constraints are chosen and committed to on the basis of the considerations mentioned in the previous section, and will depend on specific interpretive policies and aims. Now, formalization constraints give us a range of formalization functions. The intended outcome of the process of formalization is an argument in the target language provided by some specific formalization function. However, as is well accepted, in each case there may be different yet equally admissible formalizations leading to the same verdict regarding validity. It is thus not a specific formalization that one commits to, but rather some constraints on formalization. A function abiding by those constraints can then be chosen arbitrarily.

It may be that a given set of formalization constraints allows for formalizations which do not agree on the validity of a given argument. In such a case, the reasoner should strive to settle the matter by adding further formalization constraints.

Finally, we may consider some formalization meta-constraints: constraints on formalization constraints. In fact, we have already 
formulated one: The formalization function should always take a declarative sentence of the source language to a sentence of the target language. More meta-constraints can be formulated along the lines of criteria for adequate formalization discussed in the literature, that we have mentioned in $\S 2$. The following criterion is endorsed by Baumgartner and Lampert (2008):

Correspondence: The formalization of an argument should be valid iff the source argument is informally valid.

This meta-constraint employs the notion of informal validity, which requires further elaboration - especially in light of the fact that we do not assume that logical forms are already present in the informal argument pre-formalization.

Another formalization meta-constraint we may consider is the principle of univocality, by which the formalization function should take every two occurrences of the same expression in an argument in the source language to the same expression in the target language. We shall discuss this meta-constraint in the following subsection, along with the meta-constraint by which co-referring expressions in the source language should be formalized by the same expression in the target language. We tie these meta-constraints to Iacona's constraints univocality and co-reference, as a basis for a comparison of our views.

\subsection{Univocality, equivocation and co-reference}

It is customary to assume that given an argument in a source language to be formalized, the context is constant throughout. This assumption boils down to a brute force decontextualization of the source argument. Equivalently, one may assume the following formalization meta-constraint, which we shall refer to as the principle of univocality:

(PU) For any expression $e$ in the source language and any contexts $c, c^{\prime}, \mathrm{F}\left(e_{c}\right)=\mathrm{F}\left(e_{c}^{\prime}\right)$.

Iacona formulates a univocality constraint (UC) along similar lines, which he claims is often taken for granted:

(UC) Any two occurrences of the same expression in an argument must be interpreted in the same way (p. 94). 
Iacona does not endorse the univocality constraint in his definition of validity, as it is insensitive to context shifts that may occur mid-argument, deeming arguments such as $(*)$ on p. 4 as valid inadvertently. Iacona's definition of validity takes into account interpretations where context has shifted in the middle of the argument (although, notably, not in the middle of a sentence as is required by example $(*)$ ).

As it comes to formal languages used as target languages for formalization, we have assumed (UC) throughout, as it is one of the purposes of the formalization to decontextualize. Hence, in the outcome of formalization, any two occurrences of the same expression will be interpreted in the same way (to be precise, what are interpreted are expression types and not occurrences, so the univocality constraint is ingrained in the framework).

The question arises as to whether there are necessary and sufficient conditions for two occurrences of expressions (i.e. expressions in context) to be assigned the same target expression by the formalization. Iacona focuses on names, and he contends that two occurrences of names should be formalized by the same target expression if and only if they denote the same object. Let us break down this claim. Iacona holds both following principles, phrased here in our terminology:

(I) If two occurrences of expressions in the source language denote distinct objects, they should be assigned different target expressions by the formalization function.

(II) If two occurrences of expressions in the source language denote the same object, they should be assigned the same target expression by the formalization function. (p. 71)

It would be helpful to distinguish two cases, one in which the two occurrences are of the same expression in the source language, and one in which we have two occurrences of different expressions in the source language. We shall review (I) and (II) in turn for both cases.

Let us consider (I), when we have two occurrences of expressions $e_{c}$ and $e_{c^{\prime}}^{\prime}$ such that $e \neq e^{\prime}$. Assume that $e_{c}$ and $e_{c^{\prime}}^{\prime}$ denote different objects. Obviously, the formalization function should give $e_{c}$ and $e_{c^{\prime}}^{\prime}$ different values, and the following formalization constraint should be endorsed: 
(12) $\mathrm{F}\left(e_{c}\right) \neq \mathrm{F}\left(e_{c^{\prime}}^{\prime}\right)$

Now, assume that we have two occurrences $e_{c}$ and $e_{c^{\prime}}$ of the same expression $e$ that denote different objects. Since the source language can be equivocal, and the target language cannot, we need to accommodate the discrepancy by assigning to $e_{c}$ and $e_{c^{\prime}}$ different target expressions. We do this by including the formalization constraint:

(13) $\mathrm{F}\left(e_{c}\right) \neq \mathrm{F}\left(e_{c^{\prime}}\right)$

So far, we are in agreement with Iacona. Now, let us consider (II). Let $e_{c}$ and $e_{c^{\prime}}^{\prime}$ be two occurrences of distinct expressions $e$ and $e^{\prime}$. Assume that $e_{c}$ and $e_{c^{\prime}}^{\prime}$ denote the same object. Iacona would have us include the formalization constraint

(14) $\mathrm{F}\left(e_{c}\right)=\mathrm{F}\left(e_{c^{\prime}}^{\prime}\right)$

This means, for instance, that "Hesperus is Phosphorus" will be formalized e.g. by " $a=a$ " and will be determined to be a logical truth (assuming a few uncontentious formalization and semantic constraints). As a result, we have that a logical truth may be a posteriori, and further, that adequate formalization may require non-trivial knowledge that goes beyond what may be expected of a competent speaker of a language. Iacona explains this odd result by distinguishing between logical knowledge and knowledge of logical form. One may be masterful in logic, and be capable of saying for every form whether it is valid or not in a given system, but still be ignorant of the logical form of some natural language sentences (p. 80). Recall that for Iacona, logical form on its logical role is determined by truth conditions. It is not expected of a rational, competent speaker of a language to have ultimate knowledge of the truth conditions of every proposition expressed by sentences of the language. One can thus be mistaken of the adequate formalization of a sentence, and thus of its logical form.

The approach to formalization that I put forward does not agree with Iacona's results. Indeed, here is where the difference in stances towards formalization and the notion of logical form has its most straightforward implications. Formalization, on the present approach, is not a descriptive procedure that reveals a pre-existing logical form. Formalization is an interpretive endeavor, highly underdetermined, which is governed by commitments of the interpreter (or 
the reasoner). Whatever constrains the formalization of a specific locution must be expressed as a commitment on behalf of the interpreter. Principles restricting the admissible constraints are expressed as meta-commitments. Thus, the formalization function will be forced to assign the same target expression to $e_{c}$ and $e_{c^{\prime}}^{\prime}$ only if the interpreter carrying out the formalization is ready to commit to the formalization constraint $\mathrm{F}\left(e_{c}\right)=\mathrm{F}\left(e_{c^{\prime}}^{\prime}\right)$, and presumably they would do so only on the basis of the presumption that $e_{c}$ and $e_{c^{\prime}}^{\prime}$ co-refer. Logical form, on this conception, is always accessible to the reasoner carrying out the formalization-indeed, it is imposed by the formalization. Surely, there are criteria for adequate formalization, as mentioned in $\S 2$. The formalization assigned by a reasoner might be inadequate in some respects - but that will not be due to its failure to capture a pre-existing logical form unknown to the reasoner.

It may be noted that the framework of semantic constraints can manage co-reference through a semantic constraint on the target language. Assume that $\mathrm{F}\left(e_{c}\right)=\hat{e}$ and that $\mathrm{F}\left(e_{c^{\prime}}^{\prime}\right)=\hat{e}^{\prime}$.

To account for the co-reference of $e_{c}$ and $e_{c}^{\prime}$, one may include the semantic constraint

(15) $\mathrm{I}(\hat{e})=\mathrm{I}\left(\hat{e}^{\prime}\right)$

having the same effect on validity as including the aforementioned formalization constraint. Standard logical systems deny this course of action, and they account for co-reference only through the use of the same expression. The framework as has been set up does not give preference to either the semantic or the formalization constraint, and we leave aside the question whether additional considerations can decide the matter. What is pertinent to the present issue is that either the formalization or the semantic constraint must be committed to by the theorizer in order to have an effect on logical properties. I suggest that while it is permissible to commit to such constraints, there shouldn't be a general rule imposing such commitments, and so the meta-constraint (II) should be rejected.

We may still consider the case where two occurrences of the same expression denote the same object. Should these two occurrences be formalized as the same target expression? For example, when formalizing "Hesperus is Hesperus", shouldn't we restrict ourselves to formalization functions that agree on their assignment to 
the two occurrences of "Hesperus", assuming that they co-refer in their respective contexts? Any reasonable formalization, it may be contended, will be thus restricted, and so $\mathrm{a}=\mathrm{a}$ is an adequate formalization for this sentence while $\mathrm{a}=\mathrm{b}$ is not. That may be so, but again, on the present approach, forcing the formalization function to give the same target expression to the two occurrences of "Hesperus" needs to be done through committing to a formalization constraint. Here, as well, a meta-constraint forcing co-referring occurrences of the same expression to be assigned the same target expression should be rejected, as there may be cases of univocality where the theorizer would not make the appropriate commitment due to ignorance of co-reference or for any other reason.

The situation we have is as follows. We start with occurrences of expressions in context, on which we impose some patterns using formalization constraints. That two occurrences are or are not of the same expression-type may guide us in forming formalization constraints, but the acceptance of such constraints is ad hoc and depends on the specific commitments of the theorizer on the occasion of interpretation.

Iacona cites Russell and Wittgenstein as holding that in a logically ideal language, sameness of sign signifies sameness of reference and difference in sign signifies difference in reference. But here we refer back to Carnap's amendment: in a logically perfect language co-reference might be discerned from the symbols alone-provided only that appropriate rules (here: formalization and semantic constraints) have been given.

\section{Conclusion}

The present study began with Iacona's denial of the uniqueness thesis, by which there is a unique notion of logical form that fulfils both the logical and the semantic roles. I am in agreement with Iacona that there are different notions of logical form that serve the different roles. I have focused on the logical role and its connection with formalization. I proposed an alternative characterization of the logical role of logical form, by which the assignment of a logical form to a natural language sentence through formalization is not merely descriptive. Formalization is part of the interpretation and analysis of a text by a reasoner, 
interpreter or interlocutor, which ultimately serves for the evaluation of the arguments presented in the text. On this approach, logical form is not there to be discovered, but it is determined through the interpretive process. There may be criteria for the adequacy of a formalization, but the outcome is always underdetermined. The explicit statement of the rules of a formal language and of the formalization into it is then the source of the normative force of the interpretive process. Thus, in distinction to Iacona's approach, logical form is always accessible to the reasoner conducting the formalization.

On the present approach, formalization entails the explicit statement of rules and thereby of commitments on behalf of the one formalizing to the logical norms that may be associated with these rules. The framework of semantic constraints provides a formal backdrop for these commitments to be spelled out. We have seen how various principles regarding formalization can be expressed by semantic or formalization constraints or meta-constraints in this framework. In particular, we have seen that the treatment of equivocation and co-reference marks a difference between the present approach and Iacona's: while for Iacona, logical form in the cases of equivocation and co-reference might be obscure to the reasoner, on the present approach whatever is part of the logical form must be accessible through explicitly committed to constraints. ${ }^{11}$

\section{References}

Baumgartner, M. and Timm L. 2008. Adequate formalization. Synthese 164: 93-115.

Brandom, R. 1998. Making It Explicit: Reasoning, Representing, and Discursive Commitment. Cambridge, MA: Harvard University Press.

Brun, G. 2008. Formalization and the objects of logic. Erkenntnis 69 (1): 1-30. Brun, G. 2014. Reconstructing arguments. Formalization and reflective

${ }^{11}$ This paper was presented at the workshop "The Phonological Conundrum" in Jerusalem, at the VIII Workshop on Philosophical Logic in Buenos Aires and at the Logic Colloquium in Prague. I thank Andrea Bianchi, David Kashtan, Ran Lanzet, Jack Woods and an anonymous referee for comments and corrections. This research was supported by the Israel Science Foundation (grant no. 1954/17). 
equilibrium. Logical Analysis and History of Philosophy 17: 94-129.

Brun, G. 2017. Conceptual re-engineering: from explication to reflective equilibrium. Synthese, 1-30.

Brun, G. 2018. Logical expressivism, logical theory and the critique of inferences. Synthese, 1-17.

Carnap, R. 1937. The Logical Syntax of Language. London: Routledge and Kegan Paul.

Carnap, R. 1962. Logical Foundations of Probability. 2nd ed. Chicago: The University of Chicago Press.

Dutilh Novaes, C. 2012. Formal Languages in Logic: A Philosophical and Cognitive Analysis. Cambridge: Cambridge University Press.

Frege, G. 1967. Begriffsschrift. In From Frege to Gödel, edited by Jan van Heijenoort, 1-82. Cambridge MA: Harvard University Press.

Goodman, N. 1983. Fact, Fiction, and Forecast. 4th ed. Harvard University Press.

Iacona, A. 2018. Logical Form. Springer.

Kaplan, D. 1989. Demonstratives: an essay on the semantics, logic, metaphysics, and epistemology of demonstratives and other indexicals. In Themes from Kaplan, 481-566. New York: Oxford University Press.

Peregrin, J. and Svoboda, V. 2013. Criteria for logical formalization. Synthese 190(14): 2897-924.

Peregrin, J. and Svoboda, V. 2017. Reflective Equilibrium and the Principles of Logical Analysis: Understanding the Laws of Logic. Routledge.

Rawls, J. 1999. A Theory of Justice. Cambridge, MA: Harvard University Press.

Resnik, M. D. 1985. Logic: normative or descriptive? The ethics of belief or a branch of psychology? Philosophy of Science 52(2): 221-38.

Sagi, G. 2013. Logical Consequence: Between Formal and Natural Language. PhD Thesis, The Hebrew University of Jerusalem.

Sagi, G. 2014. Formality in logic: from logical terms to semantic constraints. Logique et Analyse 227: 259-76.

Sainsbury, M. 1993. Logical Forms. Oxford: Blackwell Publishers.

Steinberger, F. 2019. Three ways in which logic might be normative. Journal of Philosophy 116(1): 5-31.

Thagard, P. 1982. From the descriptive to the normative in psychology and logic. Philosophy of Science 49(1): 24-42.

Wittgenstein, L. 1922. Tractatus Logico-Philosophicus. Translated by C. K. Ogden. London: Routledge and Kegan Paul.

Woods, J. 2017. Characterizing invariance. Ergo 3: 778-807.

Woods, J. and Michaelson, E. Building character. Manuscript. 